\title{
Real Action Needed To Prevent Corona: Bangladesh Perspective
}

\author{
Md. Ridwanur Rahman \\ Professor and Former Head, Department of Medicine, Shaheed Suhrawardy Medical College, Dhaka, Bangladesh \& Chief, \\ Universal Medical Research Center, Dhaka, Bangladesh; Email: ridwanurr@yahoo.com
}

Limited community transmission of SARS CoV2 is currently underway in Bangladesh. It is still limited (controlled) due to lockdown. However, in the densely populated country one core people have left outside Dhaka in the last 3 to 5 days before declaring lockdown. Thus it is feared that it may spread further than not. In some areas the disease and the number of patients may increase. In that case the diagnosis test or test kit should be raised in that area immediately as needed; Patients need to arrange quarantine and isolation; first of all, the field hospital should be increased to provide medical care.

Since the test kit is currently lacking, it has to be supplied with need and priority. There is a shortage of test kits all over the world, so increasing the number of test kits is not possible overnight. According to the current policy of the IEDCR, it is possible to diagnose coronavirus patients in the hospital through this test kit and provide appropriate treatment.

The same applies to our Capacity Limited, America, so we can keep this community transmission limited through the wide spread of telemedicine services. It is important to remember that if disease transmission is widespread, mortality will increase in our country which may outperform other countries. If the disease detection or transmission is to increase, the lockdown period should be up to two days for incubation period (ie, 24 days after the onset of the disease). If the case is decreasing, the lockdown time may be reduced and if the number of patients is increasing before 20 days, then the lockdown should be increased. The prevalence of the disease has declined due to the lockdown in China.
Scientists still have no evidence, how long the lockdown will keep the disease under control. However, lockdown can be a 'community focal' in some areas, in some places the number of diseases and patients will increase. Increase surveillance or surveillance.

The benefit is that once the case is identified, those with mild symptoms will be advised to rest or seek treatment at home, those with moderate symptoms will remain in institutional quarantines and those with acute symptoms will be admitted to the hospital. Thus the maximum use of the limited test kit will be. To do this, it is necessary to coordinate the work and set up a command and control center of 4-5 members, who will monitor the situation at every moment and take the next decision quickly. Wealth should be utilized by analyzing cost-benefit and, above all, reducing human mortality. So there is no alternative to detection or diagnosis, surveillance or monitoring and increasing the number of test kits.

Lockdown has proved to be one of the most effective approaches in the world, though its economic impact is profound. So far the case has been detached in Dhaka and its surrounding area. This may have been because there was no test facility in other areas. Some of the people who have spread to different parts of the country from Dhaka have already been infected. They are increasing the infection locale. If 5 percent of the people adhere to the methods of controlling the disease repeatedly, then the spread of the disease will come under control.

Patients who suffer from chronic cough or shortness of breath due to coronavirus, who in medical 
terminology called ARDS, will need oxygenation support. For this, an invasive ventilator machine will be required, ie, the supply of oxygen through the tube into the body, which is very scarce in Bangladesh. These patients have the highest mortality risk.

If we cannot tell the correct number of patients to the World Health Organization and the world, we will lose credibility to them. With such low numbers and deaths, foreign organizations can keep our country locked down at the end of the epidemic to monitor the exact situation and numbers.

But according to the natural nature of the spread of the virus, the spread of the disease in this region of Bangladesh may again be less, if the lockdown can be $100 \%$ effective then a two-week lockdown is sufficient. Because the virus does not live longer and the virus will not move, move or move people.

It is our responsibility to bring these patients to the Approach Medical Facility with proper ambulance when patients are suffering from Covid19 Positive and breathing. At present, there is a shortage of acupressure facilities throughout the country, but since the Army has their capacities in the field, ventilators have been developed to improve field hospitals. These plans need to be done one to two months in advance. Because there is a shortage of ventilators all over the world now. As we have said from the beginning, our medical system is adequate, so we have failed to make a proper and realistic plan. The test kits cost about Tk 5,000 each.

Since these tests are to be done for free, the private hospital may not be interested in doing these tests. If the kit and ventilator were brought in at a cost of
Tk 1,000 crores in advance, it would have been possible to save millions for the lockdown in the future. In order to reassure people, some visible actions must be performed. For example, it is possible to reduce the rate of illness and casualty by building a large Covid Hospital, the availability of a test kit and the obligation to observe lockdowns outside Dhaka and Dhaka.

Most people in our country are now aware that the disease can happen to anyone and even cause death. But the action plan is not right. In the case of hand wash we record the world record, but $5 \%$ of them have doubts about whether they wash their hands on a regular basis. But now more than 5 percent of people are washing their hands properly for fear of coronation. If more than 5 percent of people wash their hands properly, then it will be easy to prevent many other infectious diseases, including coronas. By engaging the community it will be easy to reduce the spread of the disease. For this, people have to give accurate information. It is important to present the correct information in order to create an epidemic action plan.

This is called the Hall of Society Approach. All the government ministries and machinery have to work together to declare it as a disaster in the Hall of Government Approach. Its goal will be to reduce human death and how to reduce harm. Taking real action for him.

[Bangladesh Journal of Infectious Diseases, April 2020;7(suppl_1):S1-S2]

How to cite this article: Rahman MR. Real Action Needed to Prevent Corona: Bangladesh Perspective. Bangladesh J Infect Dis 2020;7(suppl_1):S1-S2 